\title{
A New Method to Determine Tooth Positions and Dental Arch Dimensions
}

\author{
F.P. G. M. VAN DER LINDEN
}

Department of Orthodontics, University of Nymegen, The Netherlands and Center for Human Growth and Development, The University of Michigan, Ann Arbor, Michigan 48104, USA

Dental arch width dimensions have been measured by use of certain cusp tips (MOORREES, The Dentition of the Growing Child, 1959) or points at the lingual cervical margin of the teeth (BAUME, J Dent Res 29:123, 1950) as reference points. However, cusp tips wear off, vary in number and location, and do not allow adequate transformation from the deciduous canines and molars to their successors (Fig 1). Points at the lingual cervical margin are affected by the buccolingual width of a tooth and its eruption level.

Recent technical developments involve computer graphic techniques in handling dental cast information as recorded by occlusal photographs (BIGGERSTAFF, Angle Orthodont 40:28, 1970) and the direct collection of numerical data from dental casts in a two dimensional (SAvara and Sanin, Am J Phys Anthropol 30: 315,1969 ) or three dimensional way (VAN DER LINDEN, IADR Program and Abstracts of $\mathrm{Pa}$ pers, No. 286, 1970). With both approaches the construction of more appropriate reference points can be realized.

Reference points to determine tooth position and dental arch dimensions should represent the real position, the "center" of the crown, and be independent of number of cusps, location of cusps, abrasion, and eruption level.

Additional information available on request to author. Received for publication December 6, 1971.
Depending on the anatomy of the tooth crowns, these requirements can be met by using two or four points located at the circumference of the crown (Fig 2). For incisors and canines the mesial and distal midpoints are recorded and the point halfway between these two midpoints is used as the registration point for tooth position. For the premolars and deciduous first molars the most buccal and lingual points also are used and the point halfway between the latter is calculated. The midpoint between the two halfway points is used to represent the premolars and the deciduous first molars. For permanent molars the same procedure as described for the front teeth is followed. The most buccal and lingual points on the permanent molars are frequently too far away from the middle to be used in determination of the tooth position as indicated. The same is true for the buccal and lingual fissures. Second deciduous molar crowns resemble strongly the permanent ones, and are treated as such.

The reference points advocated can be used to evaluate arch form as well as arch dimensions. Because they are expressed in $x$ and $y$ coordinates, a large number of variables can be studied.

The points discussed are only some of the reference points that can be used in dental cast analyses. For other purposes, such as the recording of the space relationships within the dental arch, the contact points must be used.

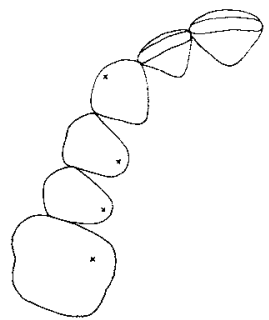

permanent teeth
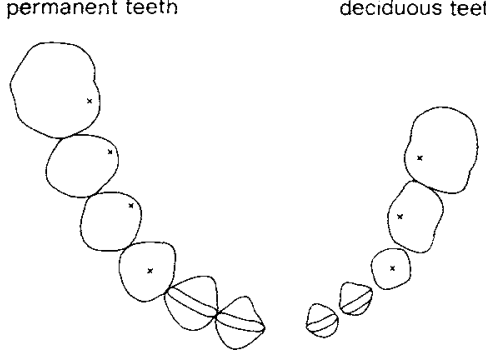

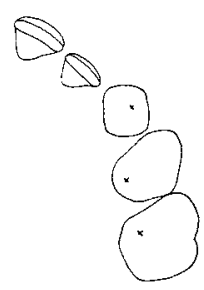

deciduous teeth
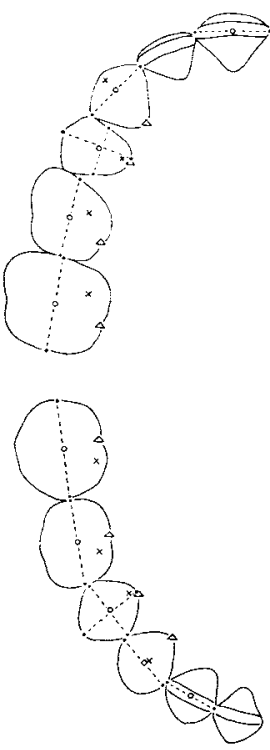

FIg 1 (left).-Differences in cusp number and location lead to variation in reference points when cusps are used $(x)$.

FIG 2 (right).-Constructed reference points (o) compared with cusp points $(x)$ and points at lingual cervical margin $(\Delta)$. 